\title{
The functional neuroanatomy of blood-injection-injury phobia: a comparison with spider phobics and healthy controls
}

\author{
X. Caseras ${ }^{1,2 *}$, V. Giampietro ${ }^{2}$, A. Lamas $^{1}$, M. Brammer ${ }^{2}$, O. Vilarroya ${ }^{1}$, S. Carmona ${ }^{1}$, M. Rovira ${ }^{3}$, \\ R. Torrubia ${ }^{1}$ and D. Mataix-Cols ${ }^{2}$ \\ ${ }^{1}$ Department of Psychiatry and Legal Medicine, Institute of Neurosciences, Autonomous University of Barcelona, Catalonia, Spain \\ ${ }^{2}$ King's College London, Institute of Psychiatry, London, UK \\ ${ }^{3}$ CRC Corporació Sanitària, Barcelona, Catalonia, Spain
}

Background. Most neuroimaging studies of specific phobia have investigated the animal subtype. The bloodinjection-injury (BII) subtype is characterized by a unique biphasic psychophysiological response, which could suggest a distinct neural substrate, but direct comparisons between phobia types are lacking.

Method. This study compared the neural responses during the presentation of phobia-specific stimuli in $12 \mathrm{BII}$ phobics, 14 spider (SP) phobics and 14 healthy controls using functional magnetic resonance imaging (fMRI).

Results. Subjective ratings showed that the experimental paradigm produced the desired symptom-specific effects. As in many previous studies, when viewing spider-related stimuli, SP phobics showed increased activation in dorsal anterior cingulate and anterior insula, compared to BII phobics and healthy controls. However, when viewing images of blood-injection-injuries, participants with BII phobia mainly showed increased activation in the thalamus and visual/attention areas (occipito-temporo-parietal cortex), compared with the other two groups. The degree of provoked anxiety and disgust by phobia-relevant images was strongly associated with activation in several common regions across the two phobia groups (thalamus, cerebellum, occipito-temporal regions) but only correlated with activation in the dorsal anterior cingulate gyrus and the anterior insula in the SP phobics.

Conclusions. These results suggest partially distinct neurobiological substrates of animal and BII phobias and support their current classification as two distinct subtypes in the DSM-IV-TR. Further research is needed to better understand the precise neurobiological mechanisms in BII phobia and particularly the fainting response.

Received 12 September 2008; Revised 15 February 2009; Accepted 18 March 2009; First published online 13 May 2009

Key words: Anxiety, fear, fMRI, specific phobia.

\section{Introduction}

The research agenda for the DSM-V emphasizes the importance of applying the findings from basic and clinical neurosciences to guide psychiatric classification (Kupfer et al. 2002). In this regard, a recently published meta-analysis of functional neuroimaging studies has suggested substantial overlap in the neural substrates of various anxiety disorders (post-traumatic stress disorder, social phobia and specific phobia) and also normal fear (Etkin \& Wager, 2007). Specifically, enhanced activation in the amygdala and insula seems to be common to normal and pathological anxiety, and has been suggested as a biomarker for these disorders. Although the results of this meta-analysis are

* Address for correspondence: X. Caseras, Ph.D., Institute of Psychiatry, Box PO69, De Crespigny Park, London SE5 8AF, UK.

(Email : x.caseras@iop.kcl.ac.uk) consistent with the existing literature in animal phobias, there has been a paucity of neuroimaging studies in other specific phobia subtypes. It is therefore unknown if all specific phobia subtypes share the same neural substrates.

The DSM-IV-TR (APA, 2000) lists four different subtypes of specific phobia [blood-injection-injury (BII), animal, situational and natural environment] that are based on differences in clinical characteristics. BII phobia is characterized by a unique physiological response to the phobic stimuli. Whereas all other specific phobias lead to an increase in arousal and peripheral activation, about $80 \%$ of people with BII phobia experience a biphasic response characterized by an initial increase in heart rate and blood pressure (sympathetic discharge), followed by a marked decrease in these parameters (parasympathetic excitation) that can easily lead to fainting (Graham et al. 1961; Thyer et al. 1985). 
Table 1. Between-group comparisons of demographic, questionnaire and subjective rating data

\begin{tabular}{lcccl}
\hline & $\begin{array}{l}\text { SP phobics } \\
(n=14) \\
\text { Mean (s.D.) }\end{array}$ & $\begin{array}{l}\text { BII phobics } \\
(n=12) \\
\text { Mean (s.D.) }\end{array}$ & $\begin{array}{l}\text { Controls } \\
(n=14) \\
\text { Mean (s.D.) }\end{array}$ & $\begin{array}{l}\text { Pairwise } \\
\text { comparison }\end{array}$ \\
\hline Gender (o), $n(\%)$ & $3(21.4 \%)$ & $3(25 \%)$ & $3(21.4 \%)$ & N.s. \\
Age (years) & $22.71(2.89)$ & $24.58(4.31)$ & $23.00(2.66)$ & N.s. \\
FSQ $^{\mathrm{a}}$ & $87.00(21.12)$ & $31.41(23.00)$ & $18.28(0.46)$ & SP $>$ BII, Control \\
MQ $^{\mathrm{a}}$ & $5.64(4.60)$ & $21.66(4.69)$ & $3.99(1.79)$ & BII $>$ SP, Control \\
N-Fear & $0.35(0.63)$ & $0.33(0.49)$ & $0.14(0.23)$ & N.S. \\
N-Disgust & $0.14(0.23)$ & $0.16(0.24)$ & $0.21(0.32)$ & N.s. \\
SP-Fear & $6.42(1.31)$ & $1.41(1.47)$ & $0.5(0.85)$ & SP $>$ BII, Control \\
SP-Disgust & $6.78(1.45)$ & $1.66(2.08)$ & $0.57(0.85)$ & SP $>$ BII, Control \\
BII-Fear & $2.57(2.72)$ & $6.25(1.91)$ & $0.57(0.51)$ & BII $>$ SP $>$ Control \\
BII-Disgust & $2.64(2.84)$ & $6.75(2.26)$ & $0.78(0.80)$ & BII $>$ SP $>$ Control \\
\hline
\end{tabular}

FSQ, Fear of Spiders Questionnaire (scoring range 18-126); MQ, Mutilation Questionnaire (scoring range 0-30); N-Fear, subjective fear rating for neutral images; N-Disgust, subjective disgust rating for neutral images; SP-Fear, subjective fear rating for images of spiders; SP-Disgust, subjective disgust rating for images of spiders; BII-Fear, subjective fear rating for blood-injection-injury images; BII-Disgust, subjective disgust rating for blood-injection-injury images ; N.S., non-significant differences.

a These scores correspond to the second administration of these questionnaires on the same day of the scan, to ensure that they remained elevated.

Whether this characteristic peripheral response in BII phobia is also associated with a distinct pattern of brain activation is unclear. In fact, the only two functional neuroimaging studies in BII phobia published to date did not show the characteristic hyperactivation in the insula or amygdala when BII phobics were presented with generally disgusting (Schienle et al. 2003) or BII-specific (Hermann et al. 2007) stimuli. However, firm conclusions cannot be drawn because direct comparisons between subtypes of specific phobia are lacking. The present study aimed to compare the neural responses to phobia-specific stimuli in BII phobics, animal phobics and healthy controls. We tested the null hypothesis that both types of phobia would have similar neural correlates (i.e. exaggerated amygdala and insula activation) when presented with their respective phobia-relevant stimuli.

\section{Method}

\section{Participants}

Participants were undergraduate students responding to an advertisement placed on the electronic notice board of the Autonomous University of Barcelona requesting volunteers to participate in ongoing studies. Interested individuals were asked to complete an online version of the Fear of Spiders Questionnaire (FSQ; Szymanski \& O'Donohue, 1995) and the Mutilation Questionnaire (MQ; Kleinknecht \& Thorndike, 1990). Both questionnaires have reported excellent psychometric properties and the ability to discriminate between phobics and non-phobics. Participants scoring above the highest quartile on one of the questionnaires and below the lowest quartile on the other were provisionally preselected as spider (SP) and BII phobics respectively. Participants scoring below the lowest quartile on both questionnaires were provisionally preselected as controls. Candidates were then interviewed by telephone and administered a brief screening questionnaire based on the Structured Clinical Interview for DSM-IV (SCID; First et al. 1995). Only participants who met diagnostic criteria for specific phobia according to DSM-IV-TR (except the control group), did not meet criteria for depression, substance abuse or dependence and psychosis, were righthanded and suitable for a functional magnetic resonance imaging (fMRI) scan were considered further. Because of the urban and non-clinical origin of the sample, the DSM-IV-TR interference criterion for specific phobia was not considered for inclusion in the SP group. To ensure sufficient severity in the BII group, we included the additional criterion of history of fainting or dizziness in situations involving medical interventions or the sight of blood. On the day of the scan, all the participants were readministered the FSQ and the MQ to ensure that their scores remained high on their respective fears or low in both questionnaires for controls (see Table 1).

A total of 45 participants were scanned. Five participants were excluded from the analysis because of fMRI acquisition problems (three participants) or because they reported having avoided looking at some pictures during the experiment (two participants). The final sample included 40 participants (14 SP, 12 BII 
and 14 controls). Participants in the phobia groups were treatment naïve.

\section{Experimental procedure}

All individuals participated in a 6-min experiment in which they viewed nine alternating picture blocks each lasting for $30 \mathrm{~s}$. Each block included 10 different phobic (SP or BII) or neutral images, each presented for $3 \mathrm{~s}$. The blocks were separated by 9-s fixation cross periods to enable the blood oxygen level dependent (BOLD) response to return to baseline levels between blocks. A total 90 images were used (30 spiders, 30 BII, 30 neutral). Pictures in each category were selected to be different in content but similar in complexity. Examples of the neutral images depicted are household objects, mushrooms, animals, birds and butterflies. We deliberately avoided using highly aversive blood-injury images to ensure that our participants would not faint in the scanner.

On arrival at the laboratory, participants were briefed on the experimental session, their written consent was obtained, and specific instructions were given to avoid looking away or closing their eyes even if they felt that the content of the pictures was highly upsetting. After the fMRI experiment, the participants were asked to rate the level of fear and disgust produced by each category of images (on a 0-10 scale) in order to validate the symptom provocation paradigm. They were also asked to complete the FSQ and the MQ and to confirm that they had not deliberately avoided looking at any image during the task.

The study received the approval of the Ethics Committee at the Autonomous University of Barcelona.

\section{Image acquisition}

Gradient-echo echoplanar images were acquired on a 1.5-T MRI system (Philips Allegra scanner) at the Hospital Sagrat Cor in Barcelona. For each of the 114 T2*-weighted whole-brain volumes obtained, 30 non-contiguous axial planes parallel to the intercommisural plane were collected with the following parameters: repetition time (TR) $3000 \mathrm{~ms}$, echo time (TE) $50 \mathrm{~ms}$, slice thickness $4 \mathrm{~mm}$, no gap, field of view (FOV) $256 \times 256 \mathrm{~mm}$, image acquisition matrix $64 \times$ $64 \times 114$. Four dummy acquisitions were also made at the beginning of each run to set longitudinal magnetization into the steady state.

\section{Statistical analysis}

Demographic data and behavioural response

Demographic data were compared across groups using ANOVAs or $\chi^{2}$ tests as required. Fear and disgust ratings were compared across groups using
ANOVAs, followed by pairwise Scheffé tests. The significance threshold was set at $p<0.05$.

\section{fMRI data analysis}

The fMRI data were analysed with software developed at the Institute of Psychiatry (ХвАм). Subject motion correction according to the methodology described by Bullmore et al. (1999a) and smoothing with a Gaussian filter [full width at half maximum (FWHM) $9.4 \mathrm{~mm}$ ] was applied. This filter size was chosen to produce effective smoothing for each voxel over itself and its nearest neighbours in three dimensions, improving the signal-to-noise ratio. Following smoothing, a general linear model (GLM)-based time-series analysis based on a previously published wavelet-based data resampling method for fMRI data (Bullmore et al. 1999 b , 2001) was applied. The method of Friman et al. (2003) was used to constrain model fits to those deemed physiologically plausible. Individual subject activation maps were transformed into standard (Talairach) space by sequential application of a rigid body and an affine transformation (Brammer et al. 1997). In the first (rigid body) stage, each subject's data were transformed on to their own high-resolution structural image. Here we assumed a change in position but not brain shape. In the second (affine) stage, the data were further transformed on to the Talairach template image and generic brain activation maps (GBAMs) were produced for each experimental condition. Hypothesis testing was carried out at the cluster level, shown to give excellent cluster-wise type I error control in structural and functional MRI analysis (Bullmore et al. 1999b). Permutation methods and median statistics were used to allow exact computation of $p$ values with minimal assumptions and the minimization of outlier effects, rather than normal theory-based inference. Furthermore, the test statistic used in this study is computed by standardizing for individual difference in residual noise before embarking on second-level, multi-subject testing using robust permutation-based methods. This allows a mixed effects approach to analysis, an approach that has been recommended recently following a detailed analysis of the validity and impact of normal theory-based inference in fMRI in a large number of subjects (Thirion et al. 2007). ANOVAs for between-groups comparisons were conducted using a permutation-based test for voxel- or cluster-wise differences as described by Bullmore et al. (1999b). In our data set, less than one false activated cluster was expected at a maximum voxel- and clusterwise $p$ value of $<0.05$ and $<0.0025$ respectively. These values were obtained directly by data-driven methods not by normal theory. The same $p$ values were applied in the whole-brain correlation analyses. 


\section{Results}

\section{Behavioural data}

As seen in Table 1, groups were similar in gender distribution $\left[\chi^{2}(2)=0.06, p=0.97\right]$ and age $[F(2,39)=$ $1.16, p=0.32]$. As expected, the SP phobia participants showed the highest score on the FSQ $[F(2,39)=58.61$, $\left.p<0.001, \eta^{2}=0.76\right]$ and BII phobics the highest on the $\operatorname{MQ}\left[F(2,39)=85.12, p<0.001, \eta^{2}=0.82\right]$.

Neutral images did not evoke any fear or disgust in any group $[F(2,39)=0.81$ and $F(2,39)=0.24$ respectively, both $p>0.05]$. Images of spiders did induce high levels of fear and disgust in SP phobics, but not in BII phobics and controls $\left[F(2,39)=93.17, \eta_{\mathrm{p}}^{2}=0.83\right.$ and $F(2,39)=66.66, \eta^{2}{ }_{\mathrm{p}}=0.78$ respectively, both $p<$ 0.001]. BII images evoked high fear and disgust levels in BII phobics, low levels in SP phobics and close to none in controls $\left[F(2,39)=27.97, \eta^{2}{ }_{\mathrm{p}}=0.60\right.$ and $F(2,39)=25.83, \eta^{2}{ }_{\mathrm{p}}=0.58$ respectively, both $\left.p<0.001\right]$ (see Table 1).

\section{fMRI results}

The activation maps for spiders and BII pictures were obtained using the neutral images as the contrast condition.

\section{$B O L D$ responses in specific phobia participants compared to controls}

When presented with images of spiders (versus neutral images), SP phobics showed greater activation than controls in the right ventrolateral prefrontal cortex [Brodmann Area (BA) 45/47] and right insula, the dorsal anterior cingulate cortex (ACC; BA24/32), the medial frontal cortex (BA6) and the cerebellum. Conversely, controls showed increased activation in the medial prefrontal cortex (BA10) and the rostral ACC (BA32) (Fig. $1 a$ and Table 2).

When viewing images of blood-injections-injuries (versus neutral images), BII phobic participants showed greater activation than controls in the thalamus bilaterally, cerebellum, fusiform gyrus (BA19/37), precuneus (BA7) and posterior cingulate gyrus (BA31). Conversely, controls showed increased activation in the occipital cortex (BA19) and the inferior/medial temporal gyrus (BA37) (Fig. $1 b$ and Table 2).

We next tested whether the two types of pictures produced similar neural responses in the healthy group. In the $\mathrm{SP}>\mathrm{BII}$ contrast, two clusters seemed to be significant, one in the cerebellum $(x=-25, y=$ $-74, \mathrm{z}=-26$ ) and one in the visual cortex (BA18, $x=21, y=-85, z=-10)$. In the BII $>$ SP contrast, a single cluster was found in the visual cortex (BA19, $x=-47, y=-63, z=-7)$. Thus, the two types of images led to almost equivalent neural responses in healthy individuals.

\section{Comparisons between phobia types}

The phobia-specific BOLD responses were studied by means of an ANOVA comparing the activation group maps of BII and SP phobics during the presentation of their respective phobia-relevant images (i.e. the group map of BII phobics during the presentation of BII images versus the group map of SP phobics during the presentation of spider pictures).

As seen in Fig. 2 and Table 3, the SP phobics showed increased activation in the medial prefrontal cortex (BA9), the dorsal ACC (BA24/32), the right inferior and ventrolateral frontal cortex (BA44/45/47) and the right anterior insula, when compared to BII phobics. Conversely, BII phobic participants showed increased activation in the occipito-parietal cortex including the fusiform gyrus and precuneus (BA17/19/36/37/39), the cerebellum extending to the inferior and middle temporal gyri (BA19/39), the medial prefrontal cortex (BA10) and the thalamus.

\section{Correlations between subjective scores and brain activation}

To further explore the neural correlates of the provoked fear/disgust in both phobia groups, wholebrain correlation analyses between subjective ratings and BOLD were carried out in each group separately. Because the fear and disgust ratings were so highly intercorrelated (Pearson's $r>0.9$ in both groups), we only report the fear results for the sake of brevity (see Supplementary material).

Although provoked fear significantly correlated with activation in some common regions across the two groups (thalamus, cerebellum, occipito-temporal regions), there were also significant correlations that seemed to be unique to each group. Most notably, subjective fear correlated with activation in the left insula and bilateral dorsal ACC in the SP phobia group only (Fig. 3).

We next statistically compared the strength of these correlations between the two phobia groups in an additional whole-brain analysis. The results confirmed that the magnitude of the association between subjective fear and BOLD signal in the left insula $(x=-43$, $\mathrm{y}=11, \mathrm{z}=0 ; p=0.008$ ) and the bilateral ACC (right $\mathrm{x}=7, \mathrm{y}=37, \mathrm{z}=7 ; p=0.005 /$ left $\mathrm{x}=-7, \mathrm{y}=44, \mathrm{z}=26$; $p=0.000002)$ was significantly greater in the SP group than in the BII group.

\section{Discussion}

This symptom provocation study represents the first attempt to directly compare the neural correlates of BII 


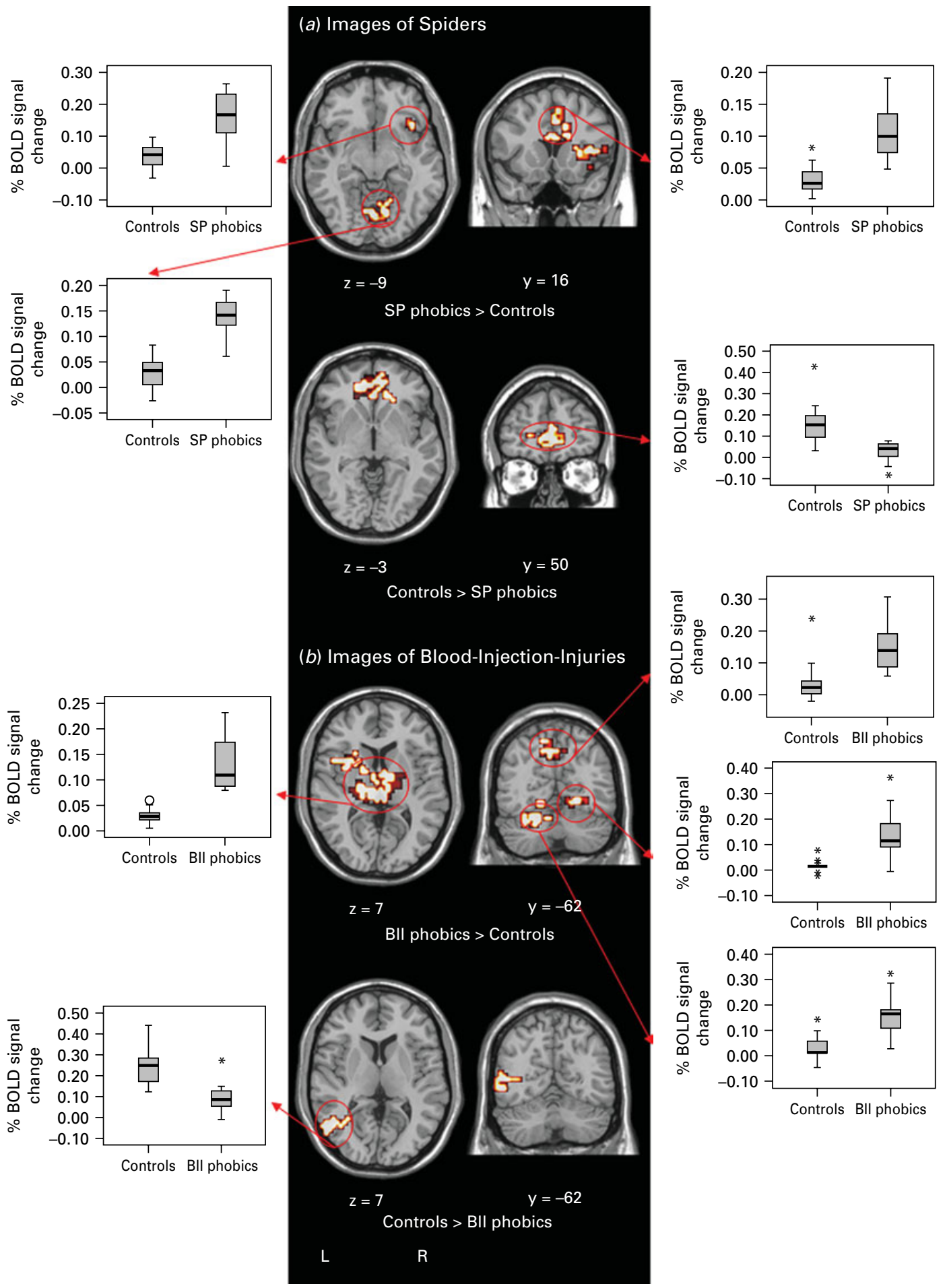

Fig. 1. Neural responses to phobia-specific symptom provocation. Regions significantly more activated (a) in spider (SP) phobics than in controls and vice versa and $(b)$ in blood-injection-injury (BII) phobics than in controls and vice versa. The functional data are superimposed on a high-resolution anatomical template using MRIcro software (www.mricro.com/mricron). L, left; $\mathrm{R}$, right.

and SP phobias and provides preliminary evidence for common as well as distinct neural substrates for these two specific phobias.
We used a robust symptom provocation procedure that successfully induced phobia subtype-specific anxiety and disgust, and replicated previous results in 
Table 2. Comparisons between phobia groups and healthy controls

\begin{tabular}{|c|c|c|c|c|c|c|}
\hline & \multirow{2}{*}{$\begin{array}{l}\text { Brodmann } \\
\text { area }\end{array}$} & \multicolumn{3}{|c|}{ Peak at } & \multirow{2}{*}{$\begin{array}{l}\text { Size in } \\
\text { voxels }\end{array}$} & \multirow[b]{2}{*}{$p$} \\
\hline & & $\mathrm{x}$ & $\mathrm{y}$ & $\mathrm{z}$ & & \\
\hline \multicolumn{7}{|l|}{ Images of spiders } \\
\hline \multicolumn{7}{|l|}{ SP phobics $>$ Controls } \\
\hline Dorsomedial prefrontal cortex/dorsal ACC (B) & $6 / 24 / 32$ & 4 & 15 & 43 & 175 & 0.0006 \\
\hline Cerebellum (B) & - & -4 & -63 & -13 & 170 & 0.0001 \\
\hline Ventrolateral prefrontal cortex/insula (R) & $45 / 47$ & 43 & 22 & 0 & 89 & 0.0007 \\
\hline \multicolumn{7}{|l|}{ Controls $>$ SP phobics } \\
\hline Medial prefrontal cortex/rostral ACC (B) & $10 / 32$ & 7 & 56 & -3 & 126 & 0.0002 \\
\hline \multicolumn{7}{|l|}{ Images of blood-injections-injuries } \\
\hline \multicolumn{7}{|l|}{ BII phobics $>$ Controls } \\
\hline Thalamus (B) & - & 0 & -19 & 3 & 237 & 0.0001 \\
\hline Cerebellum/fusiform gyrus (B) & $19 / 37$ & -4 & -70 & -10 & 173 & 0.0001 \\
\hline Precuneus/posterior cingulate cortex (B) & $7 / 31$ & 0 & -67 & 33 & 109 & 0.0008 \\
\hline Cerebellum $(\mathrm{L})$ & - & -22 & -56 & -26 & 58 & 0.001 \\
\hline \multicolumn{7}{|l|}{ Controls $>$ BII phobics } \\
\hline Occipital/inferior and middle temporal cortex (L) & $19 / 37$ & -47 & -63 & -3 & 54 & 0.002 \\
\hline
\end{tabular}

SP, Spider ; BII, blood-injection-injury ; L, left ; R, right; B, bilateral ; ACC, anterior cingulate cortex.

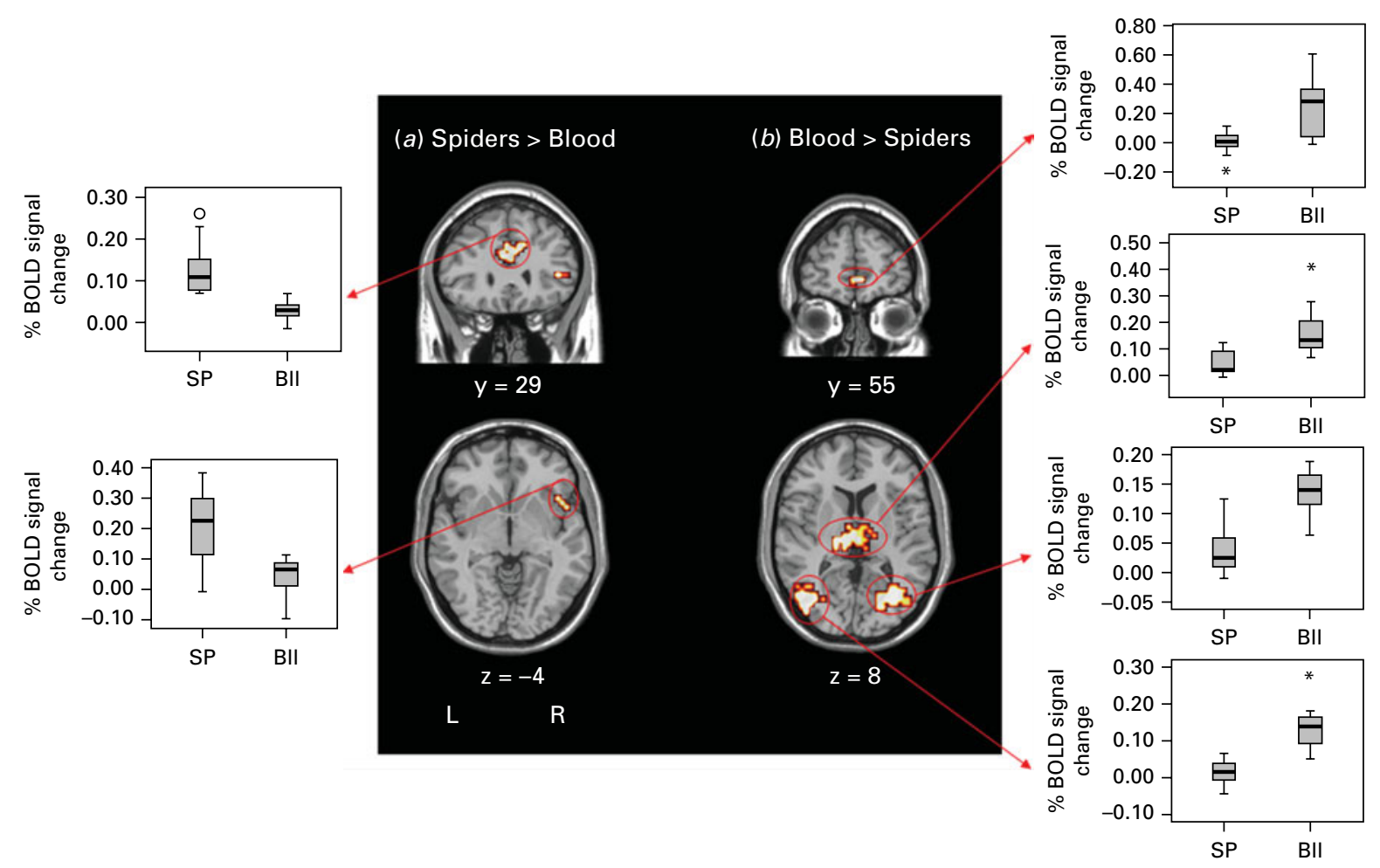

Fig. 2. Comparison of the spider (SP) and blood-injection-injury (BII) phobia groups during the presentation of their respective phobia-specific stimuli. (a) SP phobia > BII phobia ; (b) BII phobia $>$ SP phobia. The functional data are superimposed on a highresolution anatomical template using MRIcro software (www.mricro.com/mricron). L, left; R, right.

animal phobia research (Fredrikson et al. 1995; Rauch et al. 1995; Dilger et al. 2003; Straube et al. 2006b; Goossens et al. 2007a,b). Indeed, as in previous studies, SP phobics showed increased activation in the dorsal ACC bilaterally, anterior insula, inferior frontal gyrus (BA45/47) and visual cortex/cerebellum, 
Table 3. Comparisons between SP and BII phobics when presented with their specific phobia-related images

\begin{tabular}{|c|c|c|c|c|c|c|}
\hline & \multirow{2}{*}{$\begin{array}{l}\text { Brodmann } \\
\text { area }\end{array}$} & \multicolumn{3}{|c|}{ Peak at } & \multirow{2}{*}{$\begin{array}{l}\text { Size in } \\
\text { voxels }\end{array}$} & \multirow[b]{2}{*}{$p$} \\
\hline & & $x$ & $\mathrm{y}$ & $\mathrm{z}$ & & \\
\hline \multicolumn{7}{|l|}{$\mathrm{SP}>\mathrm{BII}$ phobics } \\
\hline Medial prefrontal cortex (R)/ACC (B) & $9 / 24 / 32$ & 18 & 41 & 30 & 80 & 0.001 \\
\hline Inferior/ventrolateral frontal cortex/insula (R) & $44 / 45 / 47$ & 47 & 19 & 3 & 27 & 0.003 \\
\hline \multicolumn{7}{|l|}{$\mathrm{BII}>\mathrm{SP}$ phobics } \\
\hline Occipital cortex/cerebellum (L) & 19 & -40 & -70 & 3 & 179 & 0.00005 \\
\hline $\begin{array}{l}\text { Occipital cortex/fusiform gyrus/middle and } \\
\text { inferior temporal gyrus/cerebellum (R) }\end{array}$ & $19 / 36 / 37 / 39$ & 47 & -70 & 7 & 128 & 0.0001 \\
\hline Precuneus (B) & 17 & 14 & -67 & 40 & 158 & 0.0002 \\
\hline Thalamus (B) & - & 0 & -7 & 3 & 39 & 0.0025 \\
\hline Medial prefrontal cortex (R) & 10 & 4 & 56 & -7 & 15 & 0.0027 \\
\hline
\end{tabular}

SP, Spider; BII, blood-injection-injury; L, left; R, right; B, bilateral ; ACC, anterior cingulate cortex.

The SP > BII phobics comparison shows those brain areas where SP phobics presented with images of spiders showed greater activation than BII phobics when presented with images of blood-injection-injuries. The reverse applies to the BII $>$ SP phobics comparison.
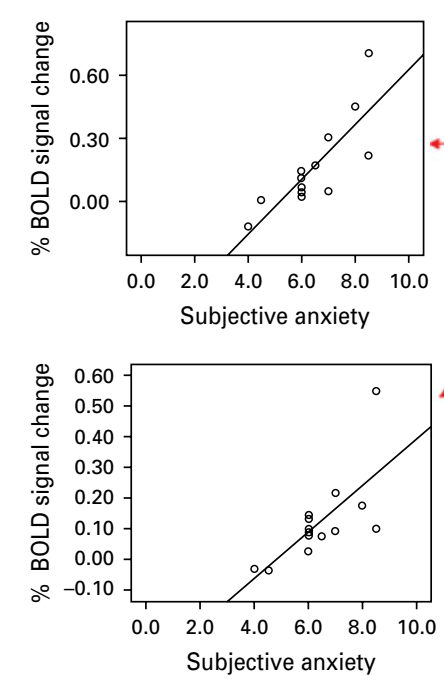

Subjective anxiety
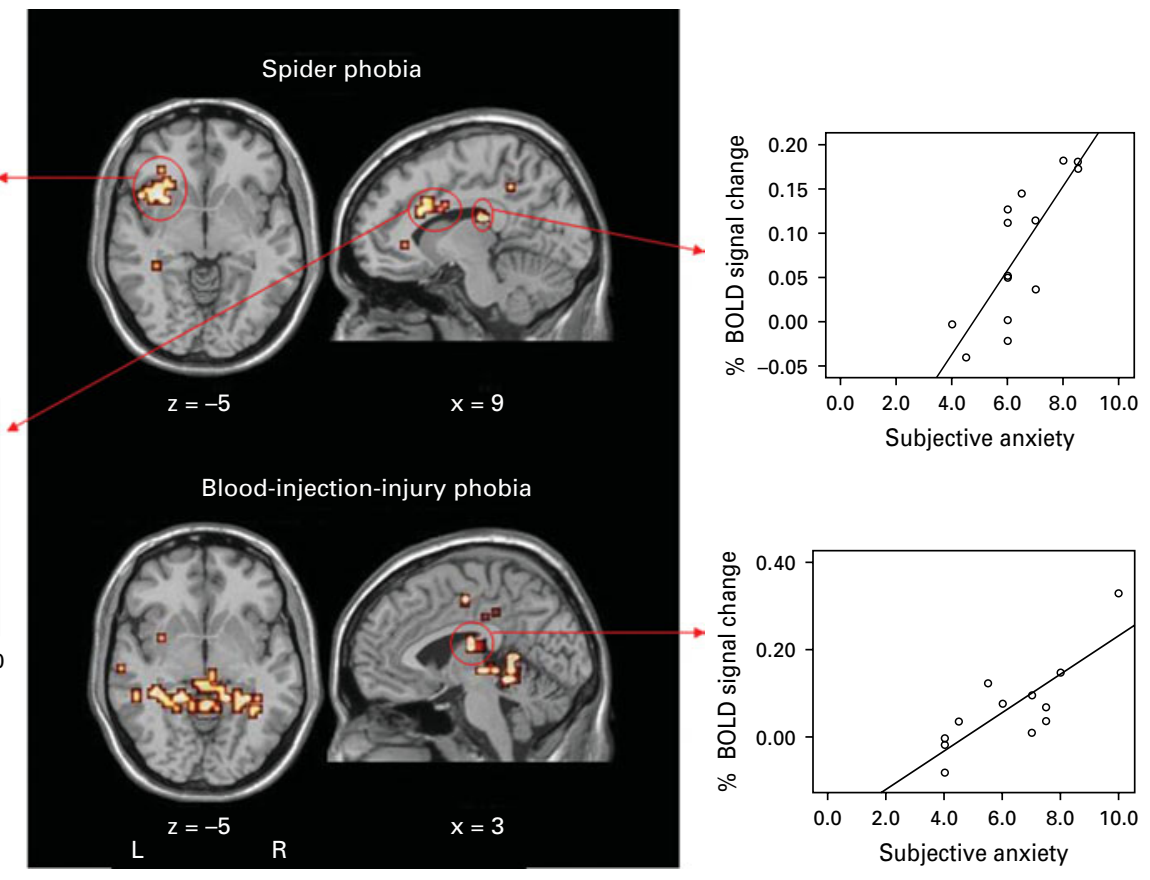

Fig. 3. Selected significant positive correlations between provoked fear and brain activation in response to phobia-specific provocation. Note that whereas provoked fear correlates with activation in the thalamus in both phobia groups, correlations in the left anterior insula and dorsal anterior cingulate gyrus are unique to the spider phobia group. The functional data are superimposed on a high-resolution anatomical template using MRIcro software (www.mricro.com/mricron). L, left; R, Right.

compared with non-phobic controls. Some of these findings were unique to SP phobia. Indeed, direct comparisons between the two phobia types during the presentation of their respective phobia-specific images showed that SP phobics had significantly increased activation in the dorsal ACC and anterior insula compared with BII phobics. In addition, as reported previously (Fredrikson et al. 1995, 1997), the SP group showed reduced activation in the medial frontal gyrus (BA10) extending posteriorly to the rostral ACC (BA32), compared with both healthy controls and BII phobics. Therefore, our symptom provocation paradigm seems to be valid to explore the neural substrates of BII phobia. 
Compared to healthy controls, BII phobics did not demonstrate increased activation in many of the brain regions previously associated with animal phobias or other anxiety disorders. Instead, increased activation was only found in bilateral occipito-parietal regions and the thalamus. Consistently, when contrasting the BOLD response between the two phobia groups during the presentation of their respective phobia-specific images, BII phobics showed greater activation in the occipito-parietal cortex and thalamus than SP phobic participants. As mentioned earlier, BII phobics also showed increased activation in the medial prefrontal cortex compared with SP, but this probably reflects reduced activation to spider phobia pictures in the SP group rather than increased activation to blood-injury pictures in the BII phobia group.

Whole-brain correlation analyses revealed that the degree of provoked fear and disgust was strongly associated with activation in several common regions across the two phobia groups (thalamus, cerebellum, occipito-temporal regions) but, confirming the above between-group comparisons, fear/disgust ratings were significantly correlated with the dorsal ACC and the anterior insula in the SP group only, thus suggesting a degree of specificity in the neural systems implicated in the two phobias.

The current results are partially consistent with two previous fMRI studies of BII phobia. Schienle et al. (2003) found that the only difference in brain region between BII phobics and healthy controls while looking at generally disgusting images was in the occipital cortex, although this study lacked a phobia-relevant condition. This finding is consistent with ours. More recently, the same group (Hermann et al. 2007) presented nine BII phobics and 10 healthy controls with phobia-relevant, generally disgusting, generally fearevoking and neutral pictures. BII phobics showed reduced dorsal and ventral medial prefrontal cortex activations compared to controls for both phobiarelevant and disgust-inducing pictures, although these results were only significant in region of interest analyses. Although the results of these studies and ours are not entirely consistent with one another, they all failed to demonstrate increased activation in many of the brain regions typically associated with animal phobias, suggesting that BII differs from those phobias in terms of their typical neural response to phobiarelevant stimuli. Whether these findings parallel the unique biphasic physiological response of this type of phobia and can teach us anything about the fainting response in BII phobia is unclear, but future studies would benefit from obtaining peripheral physiological measures at the same time as their neuroimaging data.

On a methodological note, it is important to note that all the neuroimaging studies in BII phobia conducted to date have used block designs. This strategy seems a reasonable first step when studying a disorder with unknown neural substrates such as BII phobia because it maximizes the power to detect significant activations. One obvious drawback of this approach is that it may have limited our ability to detect activations in other hypothesized brain regions that have briefer patterns of activation and are relevant to our understanding of many emotional disorders, particularly the amygdala. Others have shown that event-related designs are more sensitive than block designs to detect amygdala activation in animal phobias (Dilger et al. 2003; Straube et al. 2006a; Goossens et al. 2007a,b). A logical next step will be to use event-related designs to explore the role of the amygdala in BII phobia, compared with other phobia subtypes.

If replicated, the current results would have implications for our current understanding of the neurobiology and classification of anxiety disorders. Current neurobiological models of fear and anxiety highlight the crucial role of the amygdala and related brain regions in both normal and pathological anxiety states (e.g. LeDoux, 2003; Paulus \& Stein, 2006). Indeed, based on the results of their meta-analysis of functional neuroimaging studies, Etkin \& Wager (2007) suggested that the amygdala and the insula play a key role in normal anxious states and in all anxiety disorders. However, the current findings suggest the possibility that BII phobia may be unlike the other anxiety disorders, not only at the peripheral physiological level but also centrally in the brain. Unfortunately, neuroimaging studies do not permit any conclusions regarding the aetiology of mental disorders, but our results suggest different underlying mechanisms in these two phobia subtypes and would therefore support the current classification of BII phobia as a separate subtype of specific phobia in the DSM and ICD.

Some limitations of the present study need to be acknowledged. First, we did not record peripheral autonomic arousal during the fMRI experiment. This limited our ability to examine how the peripheral and central measures of activation co-varied across the experimental session. Second, as mentioned earlier, the use of a block design may have limited the sensitivity of our experiment to detect activation in the amygdala, a key structure in the neurobiology of anxiety and its disorders (Etkin \& Wager, 2007). Third, we did not ascertain the presence of other anxiety disorders in this sample. Fourth, whether the results will generalize to treatment-seeking samples remains to be seen, although the inclusion of treatment-naive participants can also be regarded as a strength of this study. 
In conclusion, BII and SP phobics share some common neural substrates (i.e. activation in occipitotemporal regions and the thalamus) but clearly differ regarding the involvement of other key areas involved in the processing and integration of emotional responses (i.e. insula and ACC). Further research is needed to understand the neurobiological mechanisms implicated in BII and particularly the fainting response.

\section{Acknowledgements}

This study was supported by project grant SEJ200602870 to X.C., R.T. and D.M.C. from the Ministerio de Educación y Ciencia (Spain).

\section{Declaration of Interest}

None.

\section{Note}

Supplementary material accompanies this paper on the Journal's website (http://journals.cambridge.org/ psm).

\section{References}

APA (2000). Diagnostic and Statistical Manual of Mental Disorders, 4th edn, text revision (DSM-IV-TR). American Psychiatric Association: Washington, DC.

Brammer M, Bullmore ET, Simmons A, Williams SCR, Grasby PM, Howard RJ, Woodruff PWR, Rabe-Hesketh S (1997). Generic brain activation mapping in functional magnetic resonance imaging: a nonparametric approach. Magnetic Resonance Imaging 15, 763-770.

Bullmore ET, Brammer M, Rabe-Hesketh S, Curtis V, Morris R, Williams SCR, Sharma T, McGuire PK (1999a). Methods for the diagnosis and treatment of stimulus correlated motion in generic brain activation studies using fMRI. Human Brain Mapping 7, 38-48.

Bullmore ET, Long C, Suckling J, Fadili J, Calvert GA, Zelaya F, Carpenter TA, Brammer MJ (2001). Coloured noise and computational inference in neurophysiological (fMRI) time series analysis: resampling methods in time and wavelet domains. Human Brain Mapping 12, 61-78.

Bullmore ET, Suckling J, Overmeyer S, Rabe-Hesketh S, Taylor E, Brammer MJ (1999b). Global, voxel and cluster tests, by theory and permutation, for a difference between two groups of structural MR images of the brain. IEEE Transactions on Medical Imaging 18, 32-42.

Dilger S, Straube T, Mentzel HJ, Fitzek C, Reichenbach JR, Hecht H, Krieschel S, Gutberlet I, Miltner WHR (2003). Brain activation to phobia-related pictures in spider phobic humans: an event-related functional magnetic resonance imaging study. Neuroscience Letters 348, 29-32.
Etkin A, Wager TD (2007). Functional neuroimaging of anxiety: a meta-analysis of emotional processing in PTSD, social anxiety disorder, and specific phobia. American Journal of Psychiatry 164, 1476-1488.

First MB, Spitzer RL, Gibbon M, Williams JB (1995). Structured Clinical Interview for DSM-IV Axis I Disorders, Patient Edition (SCID-I/P, Version 2.0). Biometrics Research Department, New York State Psychiatric Institute: New York, NY.

Fredrikson M, Fischer H, Wik G (1997). Cerebral blood flow during anxiety provocation. Journal of Clinical Psychiatry 58 (Suppl. 16), 16-21.

Fredrikson M, Wik G, Annas P, Ericson K, Stone-Elander S (1995). Functional neuroanatomy of visually elicited simple phobic fear: additional data and theoretical analysis. Psychophysiology 32, 43-48.

Friman O, Borga M, Lundberg P, Knutsson H (2003). Adaptive analysis of fMRI data. Neuroimage 19, 837-845.

Graham DT, Kabler JD, Lunsford L (1961). Vasovagal fainting: diphasic response. Psychosomatic Medicine 23, 493-507.

Goossens L, Schruers K, Peeters R, Griez E, Sunaert S $(2007 a)$. Visual presentation of phobic stimuli: amygdala activation via an extrageniculostriate pathway? Psychiatry Research 155, 113-120.

Goossens L, Sunaert S, Peeters R, Griez EJL, Schruers KRJ (2007b). Amygdala hyperfunction in phobic fear normalizes after exposure. Biological Psychiatry 62, $1119-1125$.

Hermann A, Schäfer A, Walter B, Stark R, Vaitl D, Schienle A (2007). Diminished medial prefrontal cortex activity in blood-injection-injury phobia. Biological Psychology 75, 124-130.

Kleinknecht RA, Thorndike RM (1990). The Mutilation Questionnaire as a predictor of blood/injury fear and fainting. Behaviour Research and Therapy 28, 429-437.

Kupfer DJ, First MB, Regier DA, eds (2002). A Research Agenda for DSM- $V$. American Psychiatric Association: Washington, DC.

LeDoux J (2003). The emotional brain, fear, and the amygdala. Cellular and Molecular Neurobiology 23, 727-738.

Paulus MP, Stein MB (2006). An insular view of anxiety. Biological Psychiatry 60, 383-387.

Rauch SL, Savage CR, Alpert NM, Miguel EC, Baer L, Breiter HC, Fischman AJ, Manzo PA, Moretti C, Jenike MA (1995). A positron emission tomographic study of simple phobic symptom provocation. Archives of General Psychiatry 52, 20-28.

Schienle A, Schäfer A, Stark R, Walter B, Kirsch P, Vailt D (2003). Disgust processing in phobia of blood-injection-injury. An fMRI study. Journal of Psychophysiology 17, 87-93.

Straube T, Glauer M, Dilger S, Mentzel HJ, Miltner WHR (2006a). Effects of cognitive-behavioral therapy on brain activation in specific phobia. Neuroimage 29, 125-135.

Straube T, Mentzel HJ, Miltner WHR (2006b). Neural mechanisms of automatic and direct processing of phobogenic stimuli in specific phobia. Biological Psychiatry 59, 162-170. 
Szymanski J, O'Donohue W (1995). Fear of Spiders Questionnaire. Journal of Behaviour Therapy and Experimental Psychiatry 26, 31-34.

Thirion B, Pinel P, Meriaux S, Roche A, Dehaene S, Poline JB (2007). Analysis of a large fMRI cohort: statistical and methodological issues for group analysis. Neuroimage 35, 105-120.

Thyer BA, Himle J, Curtis GC (1985). Blood-injuryillness phobia: a review. Journal of Clinical Psychology 41, 451-459. 